\title{
Three-dimensional numerical investigation on micro-meter droplet impact and penetration into the porous media with different velocities
}

\author{
Hongxin Deng, Yanlu Huang*, Yongqiang Yang, Shibiao Wu, and Zhiyi Chen \\ School of Mechanical and Automotive Engineering, South China University of Technology, \\ Guangzhou, China
}

\begin{abstract}
Droplets impacting and penetration into porous media is commonly seen in natural and engineering processes, in which the kinetics and capillary effect are of great importance to the lateral spreading and vertical penetration. In this study, a three-dimensional numerical simulation method was proposed to study the micro-meter droplet impact and penetration into the porous media. It is found that both the lateral spread and vertical penetration occur on the millisecond timescale and larger velocity will enhance the lateral spreading but have little influence on the penetration time and depth. The direct numerical method proposed in this study can be applied to predict the actual spreading and penetration status in the droplet-powder system and further insight into the dropletpowder interaction.
\end{abstract}

\section{Introduction}

Droplets impacting and penetration into porous media is commonly seen in natural and engineering processes such as raindrops landing on sand and binder jetting technology. In binder jetting additive manufacturing (BJAM), binder droplets are selectively deposited on the powder bed to join particles together. The processes of powder spreading, binder deposition and binder curing are repeated until the green part is finished. The green part is usually fragile and requires post-processing such as degreasing and sintering[1] to improve mechanical performance. BJ-AM is compatible with virtually any powder materials such as ceramics[2, 3], metals[4], biomaterials[5] and polymers[6].

It is of great importance to study the impact and penetration process because the lateral spreading diameter and the penetration depth have a great influence on the layer thickness and the dimensional accuracy. In binder jetting process, the droplet size is usually of micrometer sized and is comparable to the powder particle size or even smaller. Although the impact process can be captured by using high-speed imaging technology, the liquid flow, velocity and pressure field inside the powder bed is hard to study experimentally.

Numerical method has been widely applied in the modelling of droplets penetration into the porous media. Hua Tan[7] proposed a direct numerical simulation method to study the micron-sized droplet impacting on the loose powder bed and found that the large impact

${ }^{*}$ Corresponding author: yanlu@scut.edu.cn 
velocity results in a wider spread and deeper penetration. $\mathrm{Fu}[8]$ performed a 2-dimensional numerical simulation on sessile droplet spreading and penetration on porous surfaces by the VOF and CSF method. Yang[9] proposed an equivalent cylindrical mapping infiltration model to represent the porosity of the model in the two-dimensional plane and performed a simulation of printing a layer by multiple nozzles at one time.

Above all, most of these works are based on the 2-dimensional simplification or the assumption of a loosely packed powder bed, which is quite different from the real process. A veritable 3-dimensional numerical model would be quite significant to investigate the liquid flow in the porous media.

In current study, assuming all the powder particles fixed at their position and ignoring the impact force of droplets. the numerical simulation of a single micro-meter droplet impact and penetration into the closely packed powder bed with different velocities is presented.

\section{Modelling}

\subsection{Physical model}

The porous media is represented by hundreds of solid spheres. In order to achieve a higher packing density, it is common to use powder of different sizes such as bimodal[10] or trimodal powder and wide powder size distribution[11] because the small particles can fill the voids between the large particles.

For simplicity we assume that there are two types of powder particles in the powder bed, and the diameter of the large one is $50 \mu \mathrm{m}$ and the other is $36.6 \mu \mathrm{m}$. The powder particles are packed with a modified body-centered-cubic (BCC) pattern as shown in Fig. 1. The large particles are set at the vertices of a cube and tangent to each other, and the small particles are set at the center of the cube and tangent to the eight surrounding large particles. The porosity of the modified BCC packing is 0.271 . In the powder bed model shown in Fig. 1 , the number of larger particles is $8 \times 8 \times 5$ and the number of smaller particles is $7 \times 7 \times 4$ and thus the size of the powder bed models is $400 \times 400 \times 250 \mu \mathrm{m}$.

In this section, the simulation of a single spherical binder droplet with a diameter of 100 $\mu \mathrm{m}$ falling from $25 \mu \mathrm{m}$ height and impacting on the powder bed is carried out through the Fluent software. The fluid properties are listed in table1.

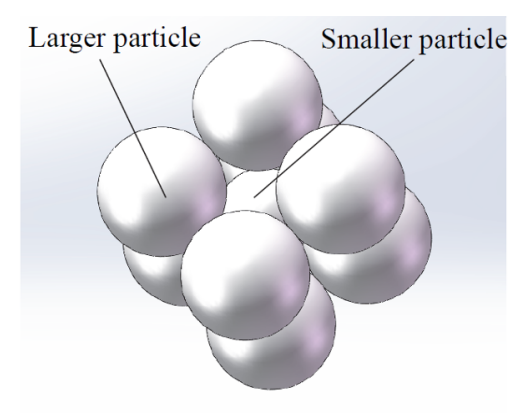

a) modified BCC packing

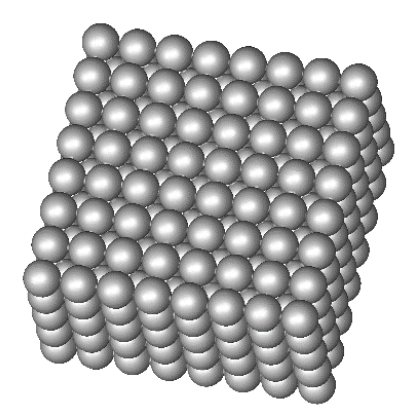

b) powder bed model

Fig. 1. The illustration of the modified BCC powder packing method and powder bed model. 
Table 1. The fluid properties.

\begin{tabular}{lll}
\hline properties & Air & Liquid \\
\hline density $\left(\mathrm{kg} / \mathrm{m}^{3}\right)$ & 1.225 & 1200 \\
Viscosity $(\mathrm{kg} / \mathrm{m}-\mathrm{s})$ & $1.79 \mathrm{e}-05$ & 0.01 \\
Contact angle & & $30^{\circ}$ \\
Surface tension $(\mathrm{N} / \mathrm{m})$ & & 0.04 \\
\hline
\end{tabular}

\subsection{Mathematical model}

In the impact and penetration process, the incompressible laminar flow obeys the continuum equation and the Navier-Stokes equation

$$
\begin{gathered}
\nabla \cdot \boldsymbol{u}=0 \\
\rho \frac{\partial \boldsymbol{u}}{\partial t}+\rho(\boldsymbol{u} \cdot \nabla) \boldsymbol{u}=-\nabla p+\mu \Delta \boldsymbol{u}+\rho \boldsymbol{g}+\boldsymbol{s}
\end{gathered}
$$

where $\boldsymbol{u}$ is velocity vector, $\rho$ is density, $p$ is pressure, $\boldsymbol{g}$ is the gravity and $\boldsymbol{s}$ is the source term due to surface tension.

Since the flow in impact and penetration process is immiscible two-phase flow, the volume of fluid (VOF) method is suitable for tracking the liquid-gas interface and the density and viscosity are dependent on the volume fraction.

$$
\begin{aligned}
& \rho=\mathrm{c} \rho_{l}+(1-c) \rho_{g} \\
& \mu=\mathrm{c} \mu_{l}+(1-c) \mu_{g}
\end{aligned}
$$

where $\mathrm{c}$ is the volume fraction of liquid in each cell, $\rho_{l}, \rho_{g}$ and $\mu_{l}, \mu_{g}$ are the density and viscosity of the liquid binder and air respectively. The surface of the powder particles is set as no-slip wall and the contact angle remains constant.

\section{Results and discussion}

a) $\mathrm{V} 1 \mathrm{~m} / \mathrm{s}$

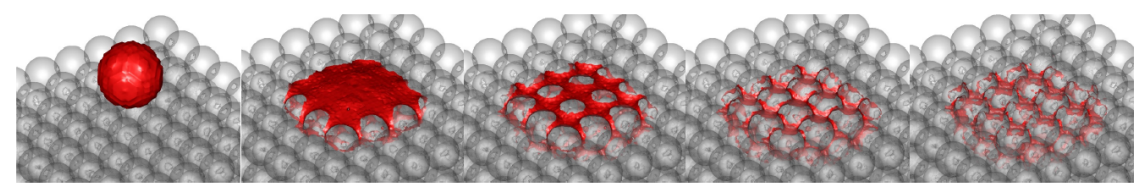

b) $\mathrm{V} 10 \mathrm{~m} / \mathrm{s}$

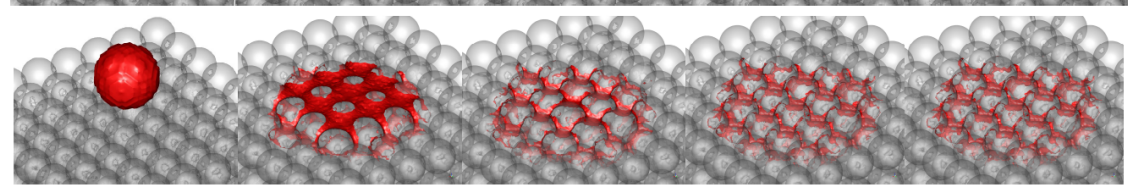

$$
\mathrm{t}=0 \mathrm{~ms} \quad \mathrm{t}=1 \mathrm{~ms} \quad \mathrm{t}=2 \mathrm{~ms} \quad \mathrm{t}=3 \mathrm{~ms} \quad \mathrm{t}=5 \mathrm{~ms}
$$

Fig. 2. Droplet impact and penetration into the powder bed with different velocities. a) the droplet velocity is $1 \mathrm{~m} / \mathrm{s}$; b) the droplet velocity is $10 \mathrm{~m} / \mathrm{s}$. 


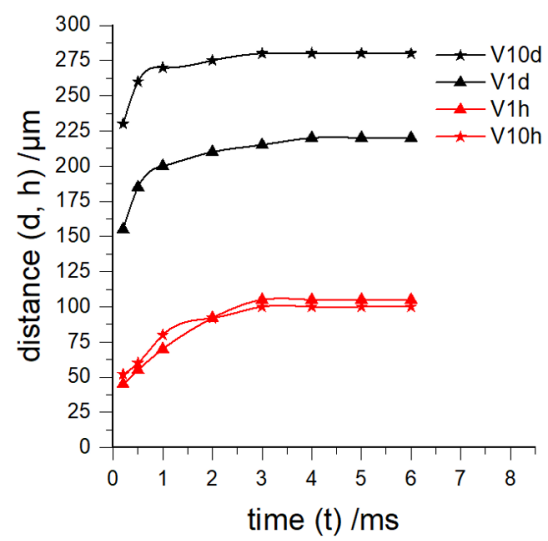

Fig. 3. the lateral spreading diameter and penetration depth under different droplet velocities. V10d: the spreading diameter when the velocity is $10 \mathrm{~m} / \mathrm{s} ; \mathrm{V} 1 \mathrm{~d}$ : the spreading diameter when the velocity is $1 \mathrm{~m} / \mathrm{s} ; \mathrm{V} 1 \mathrm{~h}$ : the penetration depth when the velocity is $1 \mathrm{~m} / \mathrm{s} ; \mathrm{V} 10 \mathrm{~h}$ : the penetration depth when the velocity is $10 \mathrm{~m} / \mathrm{s}$.

At the time $\mathrm{t}=0 \mathrm{~ms}$, the binder droplet is located $25 \mu \mathrm{m}$ above the powder bed and released with a falling velocity of $1 \mathrm{~m} / \mathrm{s}$ and $10 \mathrm{~m} / \mathrm{s}$. Fig. 2 demonstrates the binder behaviour on the powder bed after impact. It can be found that (1) the micro-meter droplet with different velocities has been fully absorbed in $5 \mathrm{~ms}$; (2) the droplet with higher velocity is absorbed more quickly, which can be seen from the contour at $1 \mathrm{~ms}$. For the higher velocity, the binder on the surface is discontinuous at $1 \mathrm{~ms}$; for the lower velocity, this discontinuous state does not appear until $2 \mathrm{~ms}$. The changing of the spreading diameter and the penetration depth is shown in Fig. 3.

From Fig. 3 it can be found that (1) both the lateral spread and vertical penetration occur on the millisecond timescale, which is in consistent with the experimental result of Holman[12]; (2) the droplet with higher velocity has a larger spreading diameter; (3) the vertical penetration depth of the droplet with different velocity is comparable. In a word, higher falling velocity has greater influence on the lateral spreading but has little influence on the vertical penetration.

The simulation result is quite different from the result gained by Hua[7]. In Hua's research, a higher velocity will result in wider spread and deeper penetration meanwhile the liquid distribution in the powder bed is segmented when the velocity is too high. It is because that in the research of Hua the powder bed model is loosely packed, in which the powder particles are not connected to each other and there is lots of void in the powder bed. The large voids between powder particles leads to too large vertical velocity transformed from kinetic energy after impact, which leads to the dispersion of binder liquid. Compared with the loosely packed powder bed proposed by Hua, the numerical closely packed powder bed model is closer to the actual situation.

The simulation result in Fig. 3 shows that droplet falling velocity has large influence on the lateral spreading diameter but little influence on the penetration depth. It is because that when the binder droplet impacts on the closely packed powder bed, most of the kinetic energy is transformed into lateral spreading velocity and only quite a small part into vertical penetration velocity. As a result, as the falling velocity increases, the lateral spreading increases rapidly and the vertical penetration is faster in the first $2 \mathrm{~ms}$. The penetration depth is an important factor in BJ-AM because the larger penetration depth strengthens the bonding between layers and improves mechanical performance. However, the fact that droplet falling velocity has little influence on the penetration depth implies that the higher 
droplet falling velocity could not help to enlarge penetration depth and strengthen layer bonding.

\section{Conclusions}

In this study, a numerical method was proposed to study micro-meter droplet impact and penetration into porous media. The porous media is represented by many fixed spheres that connect to each other in a modified BCC structure. It is found that both the lateral spread and vertical penetration of a single micro-meter droplet occur on the millisecond timescale and a higher velocity results in wider spreading diameter, but comparable penetration time and penetration depth. The direct numerical method proposed in this article can be applied to investigate the droplet-powder interaction and predict the penetration status in binder jetting process.

It has to be noted that the numerical method is based on the assumption that the powder particles are fixed at their position. However, in real droplet-powder interaction the powder particles may be moved by the droplet impact or the liquid drag. In the future, the displacement of the powder particles should be considered and a possible method is couple the CFD with the discrete element method (DEM).

\section{References}

1. Z. Marco, L.N.F. Maria, V. Maurizio, C. Ilaria, Dimensional and geometrical precision of parts produced by Binder Jetting process as affected by the anisotropic shrinkage on sintering, Additive Manufacturing, 2021; 102007.

2. N. Okpin, K. Kangmin, L. Hyunjoo, L. Hyunseung, Printability and Setting Time of CSA Cement with Na2SiO3 and Gypsum for Binder Jetting 3D Printing, Materials, 2021; 14: 2811.

3. M. Marco, B. Ruben, B. Paolo, G. Carmen, A. Raffaele, L. Nora, 3D printing of fine alumina powders by binder jetting, Journal of the European Ceramic Society, 2021; 41: 5307-5315.

4. M. Etienne, N. Arunkumar, K. Srikanth, B. Rasim, Binder jetting of "Hard-to-Weld" high gamma prime nickel-based superalloy RENÉ 108, Additive Manufacturing, 2021; 39: 101894.

5. A.J. Ho, K. Jinyoung, H. Ginam, K. DongEung, C.K. Hee, L. Hyun, K.H. Ee, K.Y. Jig, J.T. Sik, J.H. Do, 3D-printed biodegradable composite scaffolds with significantly enhanced mechanical properties via the combination of binder jetting and capillary rise infiltration process, Additive Manufacturing, 2021; 101988.

6. Y. Wang, Z. Xu, D. Wu, J. Bai, Current Status and Prospects of Polymer Powder 3D Printing Technologies, Materials, 2020; 13: 2406.

7. H. Tan, Three-dimensional simulation of micrometer-sized droplet impact and penetration into the powder bed, Chemical Engineering Science, 2016; 153: 93-107.

8. F. Fu, P. Li, K. Wang, R. Wu, Numerical Simulation of Sessile Droplet Spreading and Penetration on Porous Substrates, Langmuir, 2019; 35: 2917-2924.

9. X. Gao, W. Yang, H. Xian, X. Tu, Y. Wang, Numerical Simulation of Multi-Layer Penetration Process of Binder Droplet in 3DP Technique, Computer Modeling in Engineering \& Sciences, 2020; 124: 227-241. 
10. Y. Bai, G. Wagner, C.B. Williams, Effect of bimodal powder mixture on powder packing density and sintered density in binder jetting of metals, 2015 Annual International Solid Freeform Fabrication Symposium, 2015, pp. 758-771.

11. C. Sun, X. Tian, L. Wang, Y. Liu, C.M. Wirth, J. Günster, D. Li, Z. Jin, Effect of particle size gradation on the performance of glass-ceramic 3D printing process, Ceramics International, 2017; 43: 578-584.

12. R.K. Holman, M.J. Cima, S.A. Uhland, E. Sachs, Spreading and Infiltration of InkjetPrinted Polymer Solution Droplets on a Porous Substrate, Journal of Colloid and Interface Science, 2002; 249: 432-440. 